\title{
Effect of Sargassum horneri Extract on Circulating Bone Metabolic Markers: Supplemental Intake Has an Effect in Healthy Humans
}

\author{
Toru Matsumoto, ${ }^{a}$ Yoshinori Hokari, ${ }^{a}$ Masayuki Hashizume, ${ }^{a}$ \\ and Masayoshi Yamaguchi ${ }^{*}, b, 1$
}

${ }^{a}$ Functional Food Material Development Laboratory, Maruhachi Muramatsu, Inc., 65-1 Jonokoshi, Yaizu, Shizuoka 425-0025, Japan and ${ }^{b}$ Laboratory of Endocrinology and Molecular Metabolism, Graduate School of Nutritional Sciences, University of Shizuoka, 52-1 Yada, Shizuoka 422-8526, Japan

(Received October 23, 2007; Accepted November 12, 2007; Published online November 16, 2007)

\begin{abstract}
The extract of Sargassum horneri (S. horneri) has been shown to have an anabolic effect on bone components due to stimulating bone formation and to inhibiting bone resorption in rat femoral tissues in vitro and in vivo. This study was undertaken to determine the effect of supplemental intake of the water-solubilized $S$. horneri extract on circulating bone metabolic markers in healthy humans. Thirty-six volunteers, aged 20-60 years (16 men and 20 women), were enrolled in this study. Volunteers were divided into three groups; placebo tablet without $S$. horneri extract ( 5 men and 7 women), tablet containing S. horneri extract at $300 \mathrm{mg} / \mathrm{day}$ ( 6 men and 7 women) or $900 \mathrm{mg} / \mathrm{day}$ (5 men and 6 women). Placebo or $S$. horneri extract tablet was ingested once a day for 4 or 8 weeks. Bone-specific alkaline phosphatase and $\gamma$-carboxylated osteocalcin are serum bone markers of bone formation, and bone tartrate-resistant acid phosphatase (TRACP) and $N$-telopeptides of type I collagen are markers of bone resorption. Serum bone-specific alkaline phosphatase or $\gamma$-carboxylated osteocalcin concentration was not significantly changed after the intake of $S$. horneri extract (300 or $900 \mathrm{mg}$ /day) for 4 or 8 weeks. Serum bone TRACP activity was significantly decreased after the intake of $S$. horneri extract ( 300 or $900 \mathrm{mg} /$ day) for 8 weeks. Serum $N$-telopeptides of type I collagen concentration was significantly decreased after the intake of $S$. horneri extract ( $900 \mathrm{mg} /$ day) for 8 weeks. Meanwhile, serum calcium, inorganic phosphorus, and other biochemical findings were not changed after the intake of $S$. horneri extract ( 300 or $900 \mathrm{mg} /$ day) for 4 or 8 weeks. This study demonstrates that the prolonged intake of $S$. horneri extract has inhibitory effects on bone resorption in humans.
\end{abstract}

Key words — marine algae, Sargassum horneri, bone resorption, bone metabolism, osteoporosis

\section{INTRODUCTION}

Bone loss with increasing age induces osteoporosis, ${ }^{1-3)}$ and it may be due to increased bone resorption and decreased bone formation. Osteoporosis with a decrease in bone mass is widely recognized as a major public health problem. ${ }^{4}$ The most dramatic expression of this disease is represented by fractures of the proximal femur. Food factors may help to prevent bone loss with increasing age..$^{5-7}$ There are growing evidences that food factors may

${ }^{1}$ Present address: Division of Endocrinology and Metabolism and Lipids, Department of Medicine, Emory University School of Medicine, 1639 Pierce Driver, 1305 WMRB, Atlanta, Georgia 30322-0001, U.S.A. E-mail: yamamasa1155@yahoo.co.jp ${ }^{*}$ To whom correspondence should be addressed: Laboratory of Endocrinology and Molecular Metabolism, Graduate School of Nutritional Sciences, University of Shizuoka, 52-1 Yada, Shizuoka 422-8526, Japan have an important role in the prevention of osteoporosis. ${ }^{6,7)}$

Among various marine algae, Sargassum horneri (S. horneri) extract has an anabolic effect on bone calcification in rat femoral tissues in vitro and in vivo. ${ }^{8)}$ S. horneri extract has been demonstrated to stimulate osteoblastic bone formation and inhibit osteoclastic bone resorption in vitro using rat femoral-diaphyseal and -metaphyseal tissues. ${ }^{9,}$ 10) It is found that the active components, which have a stimulating effect on bone calcification and a suppressing effect on osteoclastic bone resorption, are different. ${ }^{11)}$ The active component in stimulating bone calcification seems to be nearby molecular weight (MW) 1000, while the active component of $S$. horneri extract in inhibiting osteoclastic cell formation is at more than MW 50000. ${ }^{11)}$ The intake of $S$. horneri extract may have a preventive effect on bone loss with increasing age., ${ }^{12)}$ and it also has 
a preventive effect on bone loss in streptozotocindiabetic rats in vivo. ${ }^{13)}$ Thus the intake of $S$. horneri extract may have a preventive effect on osteoporosis.

The present study, moreover, was undertaken to determine whether the prolonged intake of $S$. horneri extract tablet has an effect on bone metabolism in healthy humans; this was estimated using the analysis of circulating bone metabolic markers.

\section{MATERIALS AND METHODS}

\section{$S$. horneri Extracts — The marine alga $S$.} horneri was seasonally gathered from the coast at Iwate Prefecture (Japan), and was freeze-dried and powered. ${ }^{8,9)}$ The fresh marine alga gathered was homogenized in distilled water with a Physcotron homogenizer, and the homogenate was centrifuged at $5500 \times g$ in a refrigerated centrifuge for $10 \mathrm{~min}$. The $5500 \times g$ supernatant fraction was pooled for freeze-drying. Powder of the water-solubilized extract, which contains active components in stimulating bone formation and in inhibiting bone resorption, was made as tablet to use in experiments.

Experimental Procedures — Thirty-six volunteers, aged 20-60 years (16 men and 20 women), who were judged to be healthy with no abnormal liver or kidney function as assessed by standard clinical and biochemical data, were enrolled as volunteers in this study. Informed consent was obtained from all before enrollment. The intake of other foods with marine alga was prohibited during
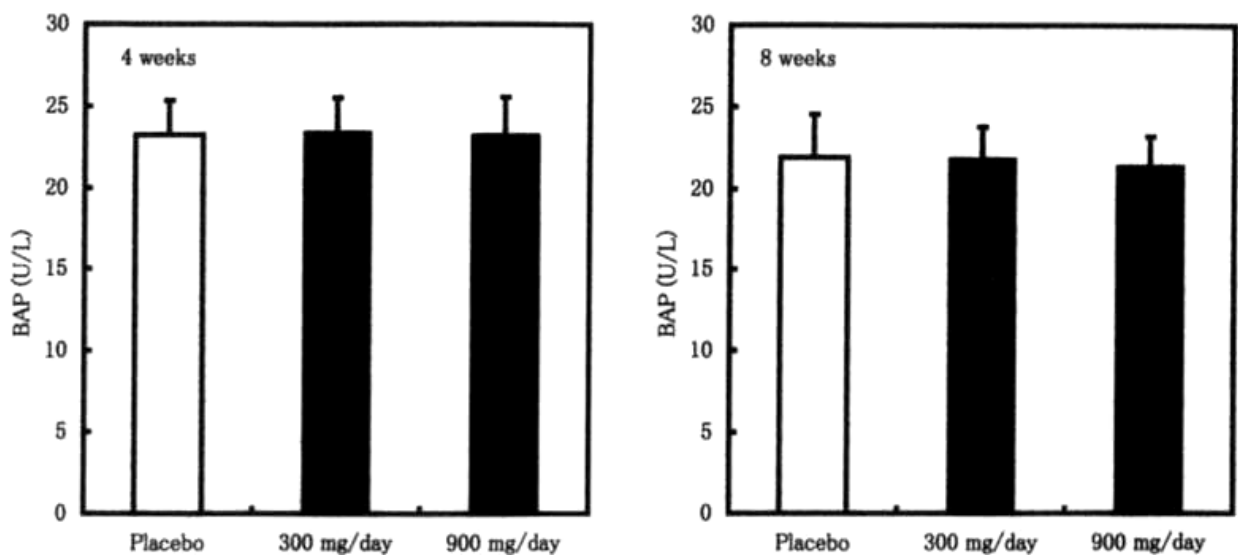

Fig. 1. Changes in Serum Bone-Specific Alkaline Phosphatase (BAP) Activity Following Intake of S. horneri Extract

S. horneri extract (placebo, 300, or $900 \mathrm{mg} /$ day) was given to volunteers once daily for 4 or 8 weeks. Thirty-six subjects were divided into three groups of 12,13, or 11 subjects for the intake of $S$. horneri extract (placebo, 300, or $900 \mathrm{mg} /$ day), respectively. Each value is the mean \pm SEM of each group subjects. There were no significant differences among the groups compared with the value obtained after placebo intake.
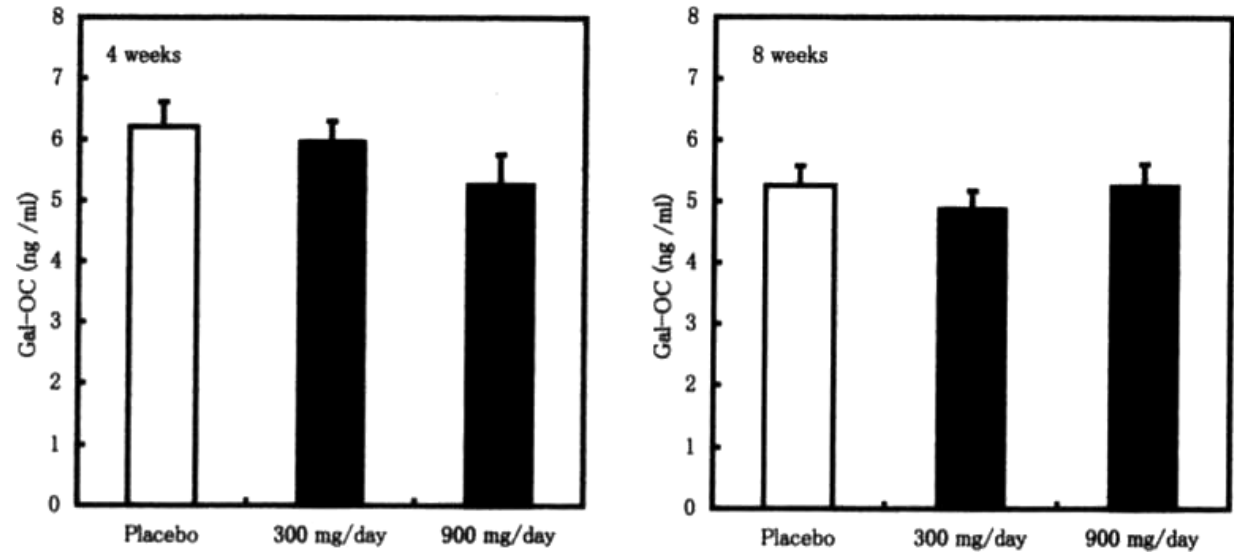

Fig. 2. Changes in Serum $\gamma$-Carboxylated Osteocalcin (Gal-OC) Concentrations Following Intake of S. horneri Extract

The procedure for the intake of $S$. horneri extract is described in Fig. 1. Each value is the mean \pm SEM of each group subjects. There were no significant differences among the groups compared with the value obtained after placebo intake. 
the experimental period.

The washout and intake periods of $S$. horneri extract tablets were 4 and 8 weeks, respectively. Volunteers were divided into three groups; placebo tablet without $S$. horneri extract (5 men and 7 women), tablet containing $S$. horneri extract at $300 \mathrm{mg} /$ day (6 men and 7 women), or $900 \mathrm{mg} /$ day (5 men and 6 women). Placebo or $S$. horneri extract tablet was sequentially given once daily for 4 or 8 weeks.

Blood samples were collected from each between 9:00 and 11:30 (morning) on the day prior to intake (control), and 4 and 8 weeks after the start of intake. Serum samples were prepared between 30 and $60 \mathrm{~min}$ after blood sampling and then stored at $-80^{\circ} \mathrm{C}$ until assayed.

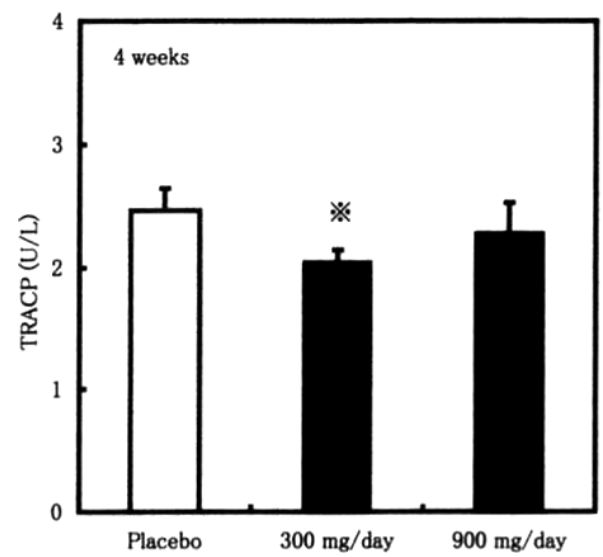

Analytical Procedures — The serum bonespecific alkaline phosphatase activity was assayed using a METRA BAP EIA kit (Quidel, San Diego, CA, U.S.A.). ${ }^{14)}$ Serum $\gamma$-carboxylated osteocalcin was assayed using a Gla type osteocalcin ( $\gamma$ carboxylated osteocalcin) EIA kit (Takara Shuzo, Shiga, Japan). ${ }^{15)}$ Serum bone tartrate-resistant acid phosphatase (TRACP) activity was assayed using a Bone TRACP Assay EIA kit (SBA Sciences, Turku, Finland). ${ }^{16)}$ Serum bone $N$-telopeptides of type I collagen was measured using an OSTEOMARK NTx Serum EIA kit (Mochida Pharmaceutical Co., Ltd., Tokyo, Japan). ${ }^{17)}$

Serum total protein, nitrogen urea, creatinine, uric acid, total cholesterol, low-density lipoprotein (LDL) cholesterol, high-density lipoprotein (HDL) cholesterol, triglyceride, total bilirubin, glutamic-

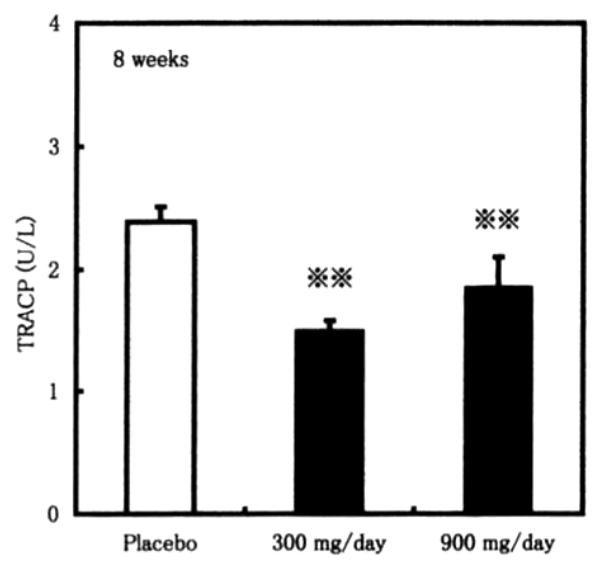

Fig. 3. Changes in Serum Bone TRACP Activity Following Intake of $S$. horneri Extract

The procedure for the intake of $S$. horneri extract is described in Fig. 1. Each value is the mean \pm SEM of each group subjects. ${ }^{*} p<0.05$ or ** $p<0.01$ compared with the value obtained after intake of placebo.
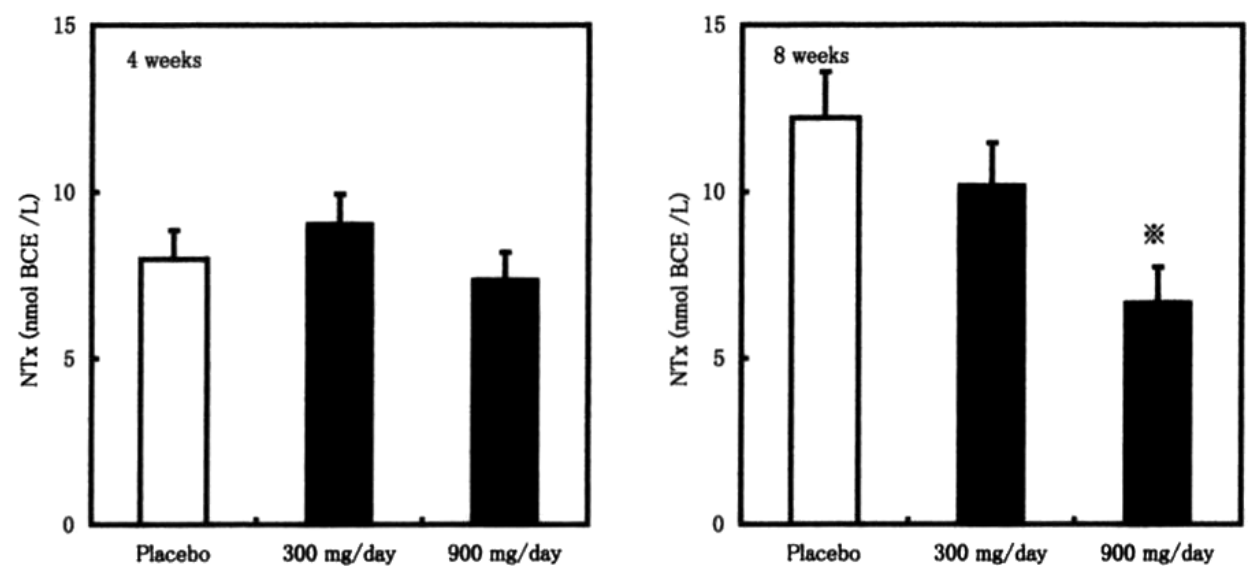

Fig. 4. Changes in Serum Concentration of $N$-Telopeptide of Type I Collagen (NTx) Following Intake of S. horneri Extract The procedure for the intake of $S$. horneri extract is described in Fig. 1. Each value is the mean \pm SEM of each group subjects. ${ }^{*} p<0.01$ compared with the value obtained after intake of placebo. 
oxaloacetic transaminase (GOT), glutamic-pyruvic transaminase (GPT), alkaline phosphatase, acid phosphatase, $\gamma$-glutamyltransferase $(\gamma$-GTP), amylase, calcium, inorganic phosphorus, and glucose levels were determined using kits.

Statistical Analysis — Differences in values after the intake of placebo and $S$. horneri extract tablet were estimated using Student's $t$-test. A paired $t$-test was used for differences in values between placebo and the intake of $S$. horneri extract tablet after each intake period. We also used multiple analysis of variance (ANOVA) to compare the treatment groups. Values of $p$ less than 0.05 were considered to represent statistically significant differences.

\section{RESULTS}

\section{Changes in Bone Metabolic Markers Following the Intake of $\boldsymbol{S}$. horneri Extract in Humans}

Thirty-six volunteers, aged 20-60 years (16 men and 20 women), were enrolled in this study. Volunteers were divided into three groups; placebo tablet without $S$. horneri extract (5 men and 7 women), tablet containing $S$. horneri extract at $300 \mathrm{mg} /$ day (6 men and 7 women), or $900 \mathrm{mg} /$ day (5 men and 6 women). Placebo or S. horneri extract tablet was sequentially given once daily for 4 or 8 weeks.
Serum bone-specific alkaline phosphatase activity (Fig. 1) or $\gamma$-carboxylated osteocalcin concentration (Fig. 2) was not significantly changed after the intake of $S$. horneri extract (300 or $900 \mathrm{mg} /$ day) for 4 or 8 weeks.

Serum bone TRACP activity was significantly decreased after the intake of $S$. horneri extract (300 mg/day) for 4 weeks (Fig. 3). TRACP activity was significantly decreased after the intake of S. horneri extract (300 or $900 \mathrm{mg} /$ day) for 8 weeks (Fig. 3).

Serum $\mathrm{N}$-telopeptides of type I collagen concentration was significantly decreased after the intake of $S$. horneri extract (900 mg/day) for 8 weeks (Fig. 4).

\section{Effects of Intake of S. horneri Extract on Other Markers}

Serum total protein, nitrogen urea, creatinine, uric acid, total cholesterol, LDL cholesterol, HDL cholesterol, triglyceride, total bilirubin, GOT, GPT, alkaline phosphatase, acid phosphatase, $\gamma$-GTP, amylase, calcium, inorganic phosphorus, and glucose levels were not significantly changed after the intake of $S$. horneri extract ( 300 or $900 \mathrm{mg} /$ day) for 8 weeks (Table 1 ).

Hematological changes (including the number of leukocytes, red blood cell, and thrombocytes) were not observed after the intake of $S$. horneri

Table 1. Serum Metabolic Findings Followng Intake of S. horneri Extract

\begin{tabular}{|c|c|c|c|}
\hline Serum level & Placebo & $300 \mathrm{mg} /$ day & $900 \mathrm{mg} /$ day \\
\hline Total protein $(\mathrm{g} / \mathrm{dl})$ & $7.4 \pm 0.45$ & $7.2 \pm 0.43$ & $7.4 \pm 0.33$ \\
\hline Urea nitrogen (mg/dl) & $12.7 \pm 3.3$ & $7.2 \pm 0.43$ & $15.1 \pm 3.4$ \\
\hline Creatinine (mg/dl) & $0.67 \pm 0.10$ & $0.72 \pm 0.18$ & $0.69 \pm 0.09$ \\
\hline Uric acid (mg/dl) & $4.8 \pm 1.3$ & $4.8 \pm 1.1$ & $4.8 \pm 1.8$ \\
\hline Total cholesterol (mg/dl) & $211.7 \pm 24.6$ & $205.0 \pm 29.0$ & $194.6 \pm 18.4$ \\
\hline LDL-cholesterol (mg/dl) & $122.4 \pm 19.0$ & $120.4 \pm 21.4$ & $111.8 \pm 17.7$ \\
\hline HDL-cholesterol (mg/dl) & $72.8 \pm 20.4$ & $67.3 \pm 14.1$ & $67.5 \pm 10.4$ \\
\hline Triglyceride (mg/dl) & $75.9 \pm 48.1$ & $67.3 \pm 14.1$ & $71.9 \pm 64.5$ \\
\hline Total bilirubin (mg/dl) & $0.58 \pm 0.24$ & $0.74 \pm 0.37$ & $0.62 \pm 0.23$ \\
\hline GOT (IU/l) & $19.1 \pm 3.2$ & $19.2 \pm 3.1$ & $22.6 \pm 3.2$ \\
\hline GPT (IU/l) & $21.0 \pm 17.0$ & $18.6 \pm 8.0$ & $20.7 \pm 25.0$ \\
\hline Alkaline phosphatase (IU/l) & $202.1 \pm 59.2$ & $187.7 \pm 44.7$ & $202.7 \pm 33.3$ \\
\hline$\gamma$-GTP (IU/l) & $24.6 \pm 18.9$ & $24.9 \pm 16.9$ & $22.0 \pm 25.2$ \\
\hline Amylase (IU/l) & $69.4 \pm 21.2$ & $73.9 \pm 18.6$ & $75.5 \pm 16.8$ \\
\hline Calcium (mg/dl) & $9.0 \pm 0.25$ & $9.0 \pm 0.37$ & $8.9 \pm 0.35$ \\
\hline Phosphorus (mg/dl) & $3.2 \pm 0.53$ & $2.9 \pm 0.29$ & $2.9 \pm 0.40$ \\
\hline Glucose (mg/dl) & $87.8 \pm 6.9$ & $90.2 \pm 9.4$ & $91.6 \pm 8.2$ \\
\hline Acid phosphatase (IU/l) & $11.3 \pm 2.4$ & $9.9 \pm 1.4$ & $10.4 \pm 2.3$ \\
\hline
\end{tabular}

S. horneri extract was given to volunteers for 8 weeks. Six thirty subjects were divided into three groups of 12,13 , or 11 subjects. Each value is the mean \pm SEM. Data were not significant as compared with that of placebo. 
extract (300 or $900 \mathrm{mg} /$ day) for 8 weeks (data not shown). Urinary biochemical findings (including calcium, inorganic phosphorus, creatinine, protein, and glucose) were not significantly changed after the intake of $S$. horneri extract ( 300 or $900 \mathrm{mg} /$ day) for 8 weeks (data not shown). Blood pressure was not significantly changed after the intake of $S$. horneri extract (300 or $900 \mathrm{mg} /$ day) for 8 weeks (data not shown).

The intake of $S$. horneri extract (300 or $900 \mathrm{mg} /$ day) for 8 weeks did not have any negative effects.

\section{DISCUSSION}

Bone loss with aging may be due to decreased bone formation and increased bone resorption. Chemical factors in food and plants may help to prevent bone loss due to increasing age. Marine alga $S$. horneri extract has been shown to have stimulatory effects on bone formation and inhibitory effects on bone resorption in rat femoral tissues in vitro and in vivo. ${ }^{8-13)}$ This study, moreover, demonstrates that the supplemental intake of $S$. horneri extract has an effect on circulating bone metabolic markers in healthy humans.

The intake of $S$. horneri extract for 8 weeks was found to have suppressive effects on serum TRACP activity and $N$-telopeptides of type I collagen level. These are bone metabolic markers that are increased in osteoclasts with bone resorption. ${ }^{18)}$ This finding suggests that the intake of $S$. horneri extract has an inhibitory effect on bone resorption in humans.

Serum bone-specific alkaline phosphatase activity or $\gamma$-carboxylated osteocalcin is bone formation markers. These proteins are produced in osteoblasts. ${ }^{19)}$ These bone formation markers were not significantly changed after the intake of $S$. horneri extract ( 300 or $900 \mathrm{mg} /$ day) for 8 weeks. A signficant increase in bone formation markers may be caused after the prolonged intake of $S$. horneri extract ( 300 or $900 \mathrm{mg} /$ day). It is speculated that the supplemental intake of $S$. horneri extract reveals suppressive effects on bone resorption, and later it exhibits stimulatory effects on bone formation in humans, thereby increasing bone mass. Presumably, the supplemental intake of $S$. horneri extract has a preventive effect on bone loss with increasing age. Further study is needed in demonstrating the effect in menopausal women.

The intake of $S$. horneri extract $(900 \mathrm{mg} /$ day $)$ for 8 weeks did not have a significant alteration in other biochemical markers for the metabolic function of organs, suggesting that the intake does not have toxic effects in humans.

In conclusion, it has been demonstrated that the intake of $S$. horneri extract has suppressive effect on serum bone resorption markers in humans.

\section{REFERENCES}

1) Nishimoto, S. K., Chang, C. -H., Gendler, E., Stryker, W. F. and Nimni, M. E. (1985) The effect of aging on bone formation in rats: biochemical and histological evidence for decreased bone formation capacity. Calcif. Tissue Int., 37, 617-624.

2) Schapira, C., Slinn, S., Sarid, M., Mokadi, S., Kabala, A. and Silibermann, M. (1995) Calcium and vitamin $\mathrm{D}$ enriched diets increase and preserve vertebral mineral content in aging laboratory rats. Bone, 16, 575-582.

3) Wild, R. A., Buchamain, J. R., Myers, C. and Demers, L. M. (1987) Declining adrenal androgen: an association with bone loss in aging women. Proc. Soc. Exp. Biol. Med., 186, 335-360.

4) Cooper, C. and Melton, J., III (1992) Epidemiology of osteoporosis. Trends Endocrinol. Metab., 3, 224229.

5) Bonjour, J. P., Schurch, M. -A. and Rizzori, R. (1996) Nutritional aspects of hip fractures. Bone, 18, 139S-144S.

6) Yamaguchi, M. (2002) Isoflavone and bone metabolism: Its cellular mechanism and preventive role in bone loss. J. Health Sci., 48, 209-222.

7) Yamaguchi, M. (2006) Regulatory mechanism of food factors in bone metabolism and prevention of osteoporosis. Yakugaku Zasshi, 126, 1117-1137.

8) Yamaguchi, M., Hachiya, S., Hiratsuka, S. and Suzuki, T. (2001) Effect of marine algae extract on bone calcification in the femoral-metaphyseal tissues of rats: Anabolic effect of Sargassum horneri. J. Health Sci., 47, 533-538.

9) Uchiyama, S. and Yamaguchi, M. (2002) Stimulatory effect of Sargassum horneri extract on bone formation in rat femoral-diaphyseal and -metaphyseal tissues in vitro. J. Health Sci., 48, 148-153.

10) Uchiyama, S. and Yamaguchi, M (2002) Inhibitory effect of marine alga Sargassum horneri extract on bone resorption in tissue culture in vitro. J. Health Sci., 48, 154-160.

11) Uchiyama, S., Hashizume, M., Hokari, Y., Nakagawa, T., Igarashi, A. and Yamaguchi, M. (2004) Characterization of active component in ma- 
rine alga Sargassum horneri extract in stimulating bone calcification in vitro. J. Health Sci., 50, 634639.

12) Uchiyama, S. and Yamaguchi, M. (2002) Anabolic effect of marine alga Sargassum horneri extract on bone components in the femoral-diaphyseal and -metaphyseal tissues of young and aged rats in vivo. J. Health Sci., 48, 325-330.

13) Uchiyama, S. and Yamaguchi, M. (2002) Preventive effect of marine alga Sargassum horneri extract on bone loss in streptozotocin-diabetic rats in vivo. J. Health Sci., 49, 149-155.

14) Koyama, N., Ohara, K., Yokota, H., Kurome, T., Katayama, M., Hino, F., Kato, I. and Aki, T. (1991) A one step sandwich enzyme immunoassay for gamma-carboxylated osteocalcin using monoclonal antibodies. J. Immunol. Methods, 139, 17-23.

15) Gomez, B., Ardakani, S., Ju, J., Jenkins, D., Cerelli, M. J., Daniloff, G. Y. and Kung, V. T. (1995) Monoclonal antibody assay for measuring bone-specific alkaline phosphatase activity in serum. Clin. Chem., 41, 1560-1566.

16) Hallen, J. M., Alatalo, S. L., Suminen, H., Cheng, S., Janckila, A. J. and Vaananen, H. K. (2000) Tartrate-resistant acid phosphatase 5b: A novel serum marker of none resorption. J. Bone Miner. Res., 15, 1337-1345.

17) Clements, J. D., Herrich, M. V., Singer, F. R. and Eyre, D. R. (1997) Evidence that serum NTx (collagen-type I N-telopeptides) can act as an immunochemical marker of bone resorption. Clin. Chem., 43, 2058-2063.

18) Asagiri, M. and Takayanagi, H. (2007) The molecular understanding of osteoclast differentiation. Bone, 40, 251-264.

19) Majeska, R. J. and Wuthier, R. E. (1975) Studies on matrix vesicles isolated from chick epiphyseal cartilage. Association of pyrophosphatase and ATPase activities with alkaline phosphatase. Biochim. Biophys. Acta, 391, 51-60. 